Honam Mathematical J. 35 (2013), No. 3, pp. 329-342

http://dx.doi.org/10.5831/HMJ.2013.35.3.329

\title{
TWO CHARACTERIZATION THEOREMS FOR LIGHTLIKE HYPERSURFACES OF A SEMI-RIEMANNIAN SPACE FORM
}

\author{
DAE Ho JiN
}

\begin{abstract}
We study lightlike hypersurfaces $M$ of a semi-Riemannian space form $\widetilde{M}(c)$ with a semi-symmetric non-metric connection whose structure vector field is tangent to $M$. Our main result is two characterization theorems for such a lightlike hypersurface.
\end{abstract}

\section{Introduction}

The theory of lightlike submanifolds is used in mathematical physics, in particular, in general relativity since lightlike submanifolds produce models of different types of horizons [10, 19]. Lightlike submanifolds are also studied in the theory of electromagnetism [4]. As for any semiRiemannian manifold there is a natural existence of lightlike subspaces, Duggal and Bejancu published their work [4] on the general theory of lightlike submanifolds to fill a gap in the study of submanifolds. Since then there has been very active study on lightlike geometry of submanifolds (see up-to date results in two books $[5,9]$ ).

Ageshe and Chafle [1] introduced the notion of a semi-symmetric nonmetric connection on a Riemannian manifold. Although now we have lightlike version of a large variety of Riemannian submanifolds, the theory of lightlike submanifolds of semi-Riemannian manifolds with semisymmetric non-metric connections has been few known. Yasar et al. [20] and Jin $[11] \sim[15]$ studied lightlike submanifolds of semi-Riemannian manifolds admitting semi-symmetric non-metric connections.

Călin proved the following result [2]: For any lightlike submanifolds $M$ of indefinite almost contact manifolds $\widetilde{M}$, if the structure vector field $\zeta$ of $\widetilde{M}$ is tangent to $M$, then it belongs to $S(T M)$. After Călin's

Received February 14, 2013. Accepted July 15, 2013.

Key words and phrases. screen quasi-conformal, lightlike hypersurface, semisymmetric non-metric connection. 
work, many earlier works $[7,8,16]$, which have been written on lightlike submanifolds of indefinite almost contact manifolds or lightlike submanifolds of semi-Riemannian manifolds admitting semi-symmetric nonmetric connections, obtained their results by using the Călin's result.

In this paper, first we prove that the afore cited Călin's result is not true for any lightlike hypersurfaces $M$ of a semi-Riemannian space form $\widetilde{M}(c)$ admitting a semi-symmetric non-metric connection (see Theorem 3.2 and its corollary). Next several authors [18] have agreed the assertion that two screen conformalities, which are called screen conformal and screen quasi-conformal, of $M$ are dependent to each other. We prove that such two screen conformalities are independent (see Theorem 3.2 and Theorem 3.3). In addition to these main results, we prove a classification theorem for Einstein lightlike hypersurfaces of a Lorentzian space form admitting a semi-symmetric non-metric connection.

\section{Semi-symmetric non-metric connection}

Let $(\widetilde{M}, \widetilde{g})$ be a semi-Riemannian manifold. A connection $\widetilde{\nabla}$ on $\widetilde{M}$ is called a semi-symmetric non-metric connection [1] if, for any vector fields $X, Y$ and $Z$ on $\widetilde{M}, \widetilde{\nabla}$ and its torsion tensor $\widetilde{T}$ satisfy

$$
\begin{gathered}
\left(\widetilde{\nabla}_{X} \widetilde{g}\right)(Y, Z)=-\pi(Y) \widetilde{g}(X, Z)-\pi(Z) \widetilde{g}(X, Y), \\
\widetilde{T}(X, Y)=\pi(Y) X-\pi(X) Y,
\end{gathered}
$$

where $\pi$ is a 1-form associated with a non-vanishing smooth vector field $\zeta$, which is called the structure vector field, by

$$
\pi(X)=\widetilde{g}(X, \zeta) .
$$

Let $(M, g)$ be a lightlike hypersurface of $\widetilde{M}$. Then the normal bundle $T M^{\perp}$ of $M$ is a subbundle of the tangent bundle $T M$ of $M$ and coincides the radical distribution $\operatorname{Rad}(T M)=T M \cap T M^{\perp}$ of $M$. Therefore there exists a complementary non-degenerate vector bundle $S(T M)$ of $\operatorname{Rad}(T M)$ in $T M$, which is called a screen distribution on $M$, such that

$$
T M=\operatorname{Rad}(T M) \oplus_{\text {orth }} S(T M),
$$

where $\oplus_{\text {orth }}$ denotes the orthogonal direct sum. We denote such a lightlike hypersurface by $M=(M, g, S(T M))$. Denote by $F(M)$ the algebra of smooth functions on $M$ and by $\Gamma(E)$ the $F(M)$ module of smooth sections of a vector bundle $E$ over $M$. It is well-known [4] that, for any null section $\xi$ of $\operatorname{Rad}(T M)$ on a coordinate neighborhood $\mathcal{U} \subset M$, 
there exists a unique null section $N$ of a unique vector bundle $\operatorname{tr}(T M)$ in $S(T M)^{\perp}$ satisfying

$$
\widetilde{g}(\xi, N)=1, \quad \widetilde{g}(N, N)=\widetilde{g}(N, X)=0, \quad \forall X \in \Gamma(S(T M)) .
$$

We call $\operatorname{tr}(T M)$ and $N$ the transversal vector bundle and the null transversal vector field of $M$ with respect to $S(T M)$ respectively. Then the tangent bundle $T \widetilde{M}$ of $\widetilde{M}$ is given by

$$
T \widetilde{M}=T M \oplus \operatorname{tr}(T M)=\{\operatorname{Rad}(T M) \oplus \operatorname{tr}(T M)\} \oplus_{\text {orth }} S(T M) .
$$

In the entire discussion of this article we shall assume that $\zeta$ to be unit spacelike vector field of $M$. Therefore $\zeta$ is tangent to $M$. In the sequel, we take $X, Y, Z \in \Gamma(T M)$ unless otherwise specified.

Let $P$ be the projection morphism of $T M$ on $S(T M)$. The local Gauss and Weingartan formulas for $M$ and $S(T M)$ are given respectively by

$$
\begin{aligned}
& \widetilde{\nabla}_{X} Y=\nabla_{X} Y+B(X, Y) N, \\
& \widetilde{\nabla}_{X} N=-A_{N} X+\tau(X) N ; \\
& \nabla_{X} P Y=\nabla_{X}^{*} P Y+C(X, P Y) \xi, \\
& \nabla_{X} \xi=-A_{\xi}^{*} X-\tau(X) \xi,
\end{aligned}
$$

where $\nabla$ and $\nabla^{*}$ are the induced linear connections on $T M$ and $S(T M)$ respectively, $B$ and $C$ are the local second fundamental forms on $T M$ and $S(T M)$ respectively, $A_{N}$ and $A_{\xi}^{*}$ are the shape operators on $T M$ and $S(T M)$ respectively, and $\tau$ is a 1-form on $T M$.

From (2.1), (2.2) and (2.6), we have

$$
\begin{gathered}
\left(\nabla_{X} g\right)(Y, Z)=-\pi(Y) g(X, Z)-\pi(Z) g(X, Y) \\
+B(X, Y) \eta(Z)+B(X, Z) \eta(Y), \\
T(X, Y)=\pi(Y) X-\pi(X) Y
\end{gathered}
$$

and $B$ is symmetric on $T M$, where $T$ is the torsion tensor with respect to the induced connection $\nabla$ of $M$ and $\eta$ is a 1-form on $T M$ such that

$$
\eta(X)=\widetilde{g}(X, N) \text {. }
$$

From the fact $B(X, Y)=\widetilde{g}\left(\widetilde{\nabla}_{X} Y, \xi\right)$, we know that $B$ is independent of the choice of a screen distribution $S(T M)$. The above two local second fundamental forms are related to their shape operators by

$$
\begin{aligned}
& g\left(A_{\xi}^{*} X, Y\right)=B(X, Y), \quad \widetilde{g}\left(A_{\xi}^{*} X, N\right)=0, \\
& g\left(A_{N} X, P Y\right)=C(X, P Y)-f g(X, P Y)-\eta(X) \pi(P Y), \\
& \widetilde{g}\left(A_{N} X, N\right)=-f \eta(X),
\end{aligned}
$$


where $f$ is the smooth function given by $f=\pi(N)$. By (2.12), we show that $A_{\xi}^{*}$ is a $S(T M)$-valued self-adjoint operator and

$$
B(X, \xi)=0, \quad A_{\xi}^{*} \xi=0 .
$$

Denote by $\widetilde{R}, R$ and $R^{*}$ the curvature tensors of the semi-symmetric non-metric connection $\widetilde{\nabla}$ on $\widetilde{M}$, the induced connection $\nabla$ on $M$ and the induced connection $\nabla^{*}$ on $S(T M)$ respectively. Using the Gauss Weingarten formulas for $M$ and $S(T M)$, we obtain the Gauss-Codazzi equations for $M$ and $S(T M)$ :

$$
\begin{gathered}
\widetilde{g}(\widetilde{R}(X, Y) Z, P W)=g(R(X, Y) Z, P W) \\
+B(X, Z) g\left(A_{N} Y, P W\right)-B(Y, Z) g\left(A_{N} X, P W\right) \\
\widetilde{g}(\widetilde{R}(X, Y) Z, \xi)=\left(\nabla_{X} B\right)(Y, Z)-\left(\nabla_{Y} B\right)(X, Z) \\
+B(Y, Z)\{\tau(X)-\pi(X)\}-B(X, Z)\{\tau(Y)-\pi(Y)\} \\
\widetilde{g}(\widetilde{R}(X, Y) Z, N)=\widetilde{g}(R(X, Y) Z, N) \\
\quad+f\{B(Y, Z) \eta(X)-B(X, Z) \eta(Y)\} \\
\widetilde{g}(\widetilde{R}(X, Y) \xi, N)=B\left(X, A_{N} Y\right)-B\left(Y, A_{N} X\right)-2 d \tau(X, Y) \\
=C\left(Y, A_{\xi}^{*} X\right)-C\left(X, A_{\xi}^{*} Y\right)-2 d \tau(X, Y), \\
g(R(X, Y) P Z, P W)=g\left(R^{*}(X, Y) P Z, P W\right) \\
+C(X, P Z) g\left(A_{\xi}^{*} Y, P W\right)-C(Y, P Z) g\left(A_{\xi}^{*} X, P W\right)
\end{gathered}
$$

(2.20) $\widetilde{g}(R(X, Y) P Z, N)$

$$
\begin{aligned}
= & \left(\nabla_{X} C\right)(Y, P Z)-\left(\nabla_{Y} C\right)(X, P Z) \\
& +C(X, P Z)\{\tau(Y)+\pi(Y)\}-C(Y, P Z)\{\tau(X)+\pi(X)\},
\end{aligned}
$$

$$
\begin{aligned}
& \widetilde{g}(\widetilde{R}(X, Y) N, P Z) \\
& =g\left(-\nabla_{X}\left(A_{N} Y\right)+\nabla_{Y}\left(A_{N} X\right)+A_{N}[X, Y], P Z\right) \\
& -\tau(Y) g\left(A_{N} X, P Z\right)+\tau(X) g\left(A_{N} Y, P Z\right),
\end{aligned}
$$

$$
\begin{aligned}
& g(R(X, Y) \xi, P Z) \\
& =g\left(-\nabla_{X}^{*}\left(A_{\xi}^{*} Y\right)+\nabla_{Y}^{*}\left(A_{\xi}^{*} X\right)+A_{\xi}^{*}[X, Y], P Z\right) \\
& \quad+\tau(Y) g\left(A_{\xi}^{*} X, P Z\right)-\tau(X) g\left(A_{\xi}^{*} Y, P Z\right) .
\end{aligned}
$$


A complete simply connected semi-Riemannian manifold $\widetilde{M}$ of constant curvature $c$ is called a semi-Riemannian space form and denote it by $\widetilde{M}(c)$. In this case, the curvature tensor $\widetilde{R}$ of $\widetilde{M}(c)$ is given by

$$
\widetilde{R}(X, Y) Z=c\{\widetilde{g}(Y, Z) X-\widetilde{g}(X, Z) Y\},
$$

for all $X, Y, Z \in \Gamma(T \widetilde{M})$.

\section{Two characterization theorems}

Lemma $3.1[11] \sim[14]$. Let $M$ be a lightlike hypersurface of a semiRiemannian manifold $\widetilde{M}$ admitting a semi-symmetric non-metric connection. If the structure vector field $\zeta$ is tangent to $M$, then $\zeta$ satisfies

$$
B(X, \zeta)=\pi\left(A_{\xi}^{*} X\right)=0 .
$$

Proof. From the two representations of (2.18), we obtain

$$
B\left(X, A_{N} Y\right)-B\left(Y, A_{N} X\right)=C\left(Y, A_{\xi}^{*} X\right)-C\left(X, A_{\xi}^{*} Y\right) .
$$

Substituting (2.12) and (2.13) into this equation, we get

$$
\pi\left(A_{\xi}^{*} X\right) \eta(Y)=\pi\left(A_{\xi}^{*} Y\right) \eta(X) .
$$

Replacing $Y$ by $\xi$ to this and using $(2.14)_{2}$, we have (3.1).

Definition 1. A lightlike hypersurface $M$ of a semi-Riemannian manifold $\widetilde{M}$ admitting a semi-symmetric non-metric connection is called screen quasi-conformal [18] if $B$ and $C$ satisfy

$$
C(X, P Y)=\varphi B(X, Y)+\eta(X) \pi(P Y),
$$

where $\varphi$ is a non-vanishing function on a neighborhood $\mathcal{U}$ in $M$.

From (2.12) and (2.13), we show that a necessary and sufficient condition for $M$ to be screen quasi-conformal is

$$
A_{N} X=\varphi A_{\xi}^{*} X-f X .
$$

Theorem 3.2. Let $M$ be a screen quasi-conformal lightlike hypersurface of a semi-Riemannian space form $\widetilde{M}(c)$ admitting a semi-symmetric non-metric connection. If $\zeta$ is tangent to $M$ but it does not belong to $S(T M)$, then $c=1$.

Proof. Applying $\nabla_{Y}$ to (3.3), we have

$$
\nabla_{X}\left(A_{N} Y\right)=X[\varphi] A_{\xi}^{*} Y+\varphi \nabla_{X}\left(A_{\xi}^{*} Y\right)-X[f] Y-f \nabla_{X} Y .
$$


Substituting this into (2.21) and using (2.11) (2.13) and (2.22), we have

$$
\begin{aligned}
\widetilde{g}(\widetilde{R}(X, Y) N, P Z)-\varphi \widetilde{g}(\widetilde{R}(X, Y) \xi, P Z) \\
=\{Y[\varphi]-2 \varphi \tau(Y)\} B(X, P Z) \\
\quad-\{X[\varphi]-2 \varphi \tau(X)\} B(Y, P Z) \\
+\{X[f]-f \tau(X)-f \pi(X)\} g(Y, P Z) \\
\quad-\{Y[f]-f \tau(Y)-f \pi(Y)\} g(X, P Z) .
\end{aligned}
$$

Substituting (2.23) into the last equation and using (2.14), we get

$$
\begin{aligned}
\{X[\varphi] & -2 \varphi \tau(X)\} B(Y, Z) \\
& -\{Y[\varphi]-2 \varphi \tau(Y)\} B(X, Z) \\
=\{X[f]-f \pi(X)-f \tau(X)+c \eta(X)\} g(Y, Z) & \quad-\{Y[f]-f \pi(Y)-f \tau(Y)+c \eta(Y)\} g(X, Z) .
\end{aligned}
$$

Taking $X=Z=\zeta$ and $Y=\xi$ to this equation and using (3.1), we have

$$
\xi[f]-f \tau(\xi)+c=0 .
$$

On the other hand, substituting (2.23) into (2.16), we have

$$
\begin{aligned}
& \left(\nabla_{X} B\right)(Y, Z)-\left(\nabla_{Y} B\right)(X, Z) \\
& \quad=B(Y, Z)\{\pi(X)-\tau(X)\}-B(X, Z)\{\pi(Y)-\tau(Y)\} .
\end{aligned}
$$

Applying $\widetilde{\nabla}_{X}$ to $\eta(Y)=\widetilde{g}(Y, N)$ and using (2.1), we have

$$
\begin{aligned}
X(\eta(Y))= & -\pi(Y) \eta(X)-f g(X, Y)+\widetilde{g}\left(\nabla_{X} Y, N\right) \\
& -g\left(A_{N} X, Y\right)+\tau(X) \eta(Y) .
\end{aligned}
$$

Substituting this into the right term of the following equation

$$
2 d \eta(X, Y)=X(\eta(Y))-Y(\eta(X))-\eta([X, Y])
$$

and using (2.11), (3.3) and the fact $A_{\xi}^{*}$ is self-adjoint, we get

$$
2 d \eta(X, Y)=\tau(X) \eta(Y)-\tau(Y) \eta(X) .
$$

Substituting (2.23) into (2.17), we obtain

$$
\begin{array}{r}
\widetilde{g}(R(X, Y) P Z, N)=c\{g(Y, P Z) \eta(X)-g(X, P Z) \eta(Y)\} \\
+f\{B(X, P Z) \eta(Y)-B(Y, P Z) \eta(X)\} .
\end{array}
$$

Comparing this equation and (2.20), we get

$$
\begin{gathered}
(3.8)\{c g(Y, P Z)-f B(Y, P Z)\} \eta(X)-\{c g(X, P Z)-f B(X, P Z)\} \eta(Y) \\
=\left(\nabla_{X} C\right)(Y, P Z)-\left(\nabla_{Y} C\right)(X, P Z)+C(X, P Z)\{\pi(Y)+\tau(Y)\} \\
-C(Y, P Z)\{\pi(X)+\tau(X)\} .
\end{gathered}
$$


Applying $\nabla_{X}$ to $C(Y, P Z)=\varphi B(Y, P Z)+\eta(Y) \pi(P Z)$, we have

$$
\begin{aligned}
& \left(\nabla_{X} C\right)(Y, P Z)=X[\varphi] B(Y, P Z)+\varphi\left(\nabla_{X} B\right)(Y, P Z) \\
& +\left\{X(\eta(Y))-\eta\left(\nabla_{X} Y\right)\right\} \pi(P Z)+\eta(Y)\left\{X(\pi(P Z))-\pi\left(\nabla_{X}^{*} P Z\right)\right\} .
\end{aligned}
$$

Substituting this into (3.8) and using (3.2), (3.4), (3.6) and (3.7), we get

$$
\begin{aligned}
& f\{\eta(Y) B(X, P Z)-\eta(X) B(Y, P Z)\} \\
& =\{X[f]-f \pi(X)-f \tau(X)\} g(Y, P Z) \\
& \quad-\{Y[f]-f \pi(Y)-f \tau(Y)\} g(X, P Z) \\
& +\eta(Y)\left\{X(\pi(P Z))-\pi\left(\nabla_{X}^{*} P Z\right)\right\} \\
& \quad-\eta(X)\left\{Y(\pi(P Z))-\pi\left(\nabla_{Y}^{*} P Z\right)\right\} .
\end{aligned}
$$

Applying $\nabla_{X}$ to $\pi(P Z)=g(\zeta, P Z)$ and using (2.10) and (3.1), we have

$$
\begin{aligned}
& X(\pi(P Z))-\pi\left(\nabla_{X}^{*} P Z\right) \\
& =-g(X, P Z)-\pi(X) \pi(P Z)+f B(X, P Z)+g\left(\nabla_{X} \zeta, P Z\right) .
\end{aligned}
$$

Substituting this equation into (3.9), we obtain

$$
\begin{aligned}
& \{X[f]-f \pi(X)-f \tau(X)\} g(Y, P Z) \\
& \quad-\{Y[f]-f \pi(Y)-f \tau(Y)\} g(X, P Z) \\
& +\eta(X)\left\{g(Y, P Z)+\pi(Y) \pi(P Z)-g\left(\nabla_{Y} \zeta, P Z\right)\right\} \\
& \quad-\eta(Y)\left\{g(X, P Z)+\pi(X) \pi(P Z)-g\left(\nabla_{X} \zeta, P Z\right)\right\}=0 .
\end{aligned}
$$

Applying $\nabla_{X}$ to $g(\zeta, \zeta)=1$ and using (2.10) and (3.1), we have

$$
g\left(\nabla_{X} \zeta, \zeta\right)=\pi(X)
$$

Taking $X=\xi$ and $Y=Z=\zeta$ to (3.10) and using (3.11), we get

$$
\xi[f]-f \tau(\xi)+1=0 .
$$

From this result and (3.5), we show that $c=1$.

Corollary 1. There exist no screen quasi-conformal lightlike hypersurfaces $M$ of a semi-Riemannian space form $\widetilde{M}(c)$ admitting a semisymmetric non-metric connection such that $\zeta$ belongs to $S(T M)$.

Proof. If $\zeta$ belongs to $S(T M)$, then $f=\widetilde{g}(\zeta, N)=0$. It follows from (3.12) that $1=0$. It is a contradiction. Thus there exist no screen quasi-conformal lightlike hypersurfaces $M$ of a semi-Riemannian space form $\widetilde{M}(c)$ admitting a semi-symmetric non-metric connection such that $\zeta$ belongs to $S(T M)$.

Remark 1. For any lightlike submanifolds $M$ of indefinite almost contact manifolds $\widetilde{M}$ such that the structure vector field $\zeta$ of $\widetilde{M}$ is tangent 
to $M$, if $\zeta$ belongs to $\operatorname{Rad}(T M)$, then $\zeta$ is decompose as $\zeta=a \xi$ and $a \neq 0$. Using this, we have $1=\widetilde{g}(\zeta, \zeta)=a^{2} \widetilde{g}(\xi, \xi)=0$. It is a contradiction. Thus $\zeta$ does not belong to $\operatorname{Rad}(T M)$. This enables one to choose a screen distribution $S(T M)$ which contains $\zeta$. Although $S(T M)$ is not unique, it is canonically isomorphic to the factor vector bundle $S(T M)^{\sharp}=T M / \operatorname{Rad}(T M)[17]$. Thus all screen distributions are mutually isomorphic. This implies that if $\zeta$ is tangent to $M$, then it belongs to $S(T M)$. Călin [2] proved this result. Duggal and Sahin also proved this result (see p.318 - 319 of [9]). After Călin's work, many earlier works $[7,8,16]$, which have been written on lightlike submanifolds of indefinite almost contact manifolds or lightlike submanifolds of semi-Riemannian manifolds admitting semi-symmetric non-metric connections, obtained their results by using the afore cited Călin's result. However, we regret to indicate that Călin's result is not true for any lightlike hypersurfaces $M$ of a semi-Riemannian space form $\widetilde{M}(c)$ admitting a semi-symmetric non-metric connection by Theorem 3.2 and its corollary.

Definition 2. A lightlike hypersurface $M$ of a semi-Riemannian manifold $\widetilde{M}$ admitting a semi-symmetric non-metric connection is screen conformal $[5,6,9]$ if the second fundamental forms $B$ and $C$ satisfy

$$
C(X, P Y)=\varphi B(X, Y),
$$

where $\varphi$ is a non-vanishing function on a neighborhood $\mathcal{U}$ in $M$.

Theorem 3.3. Let $M$ be a lightlike hypersurface of a semi-Riemannian space form $\widetilde{M}(c)$ admitting a semi-symmetric non-metric connection such that $\zeta$ is tangent to $M$. If $M$ is screen conformal, then $c=0$.

Proof. Applying $\nabla_{X}$ to $C(Y, P Z)=\varphi B(Y, P Z)$, we have

$$
\left(\nabla_{X} C\right)(Y, P Z)=X[\varphi] B(Y, P Z)+\varphi\left(\nabla_{X} B\right)(Y, P Z) .
$$

Substituting this equation into (2.20) and using (3.6), we have

$$
\begin{aligned}
& \widetilde{g}(R(X, Y) P Z, N) \\
& =\{X[\varphi]-2 \varphi \tau(X)\} B(Y, P Z)-\{Y[\varphi]-2 \varphi \tau(Y)\} B(X, P Z) .
\end{aligned}
$$

Substituting this equation and (2.23) into (2.17), we get

$$
\begin{aligned}
c\{g(Y, P Z) \eta(X)-g(X, P Z) \eta(Y)\} & \\
=\{X[\varphi]-2 \varphi \tau(X)+f \eta(X)\} B(Y, P Z) & \\
& -\{Y[\varphi]-2 \varphi \tau(Y)+f \eta(Y)\} B(X, P Z) .
\end{aligned}
$$

Taking $X=\xi$ and $Y=Z=\zeta$ to this and using (3.1), we have $c=0$. 
Jin [12] proved the following result: Under the same assumption in Theorem 3.5, if $M$ is screen conformal and $\tau=0$, then $c=0$.

Remark 2. From Theorem 3.2 and Theorem 3.3, we show that the two screen conformalities, which are called screen conformal and screen quasi-conformal, of $M$ are not mutually dependent to each other but not mutually independent.

\section{Einstein lightlike hypersurfaces}

Let $\widetilde{R i c}$ be the Ricci curvature tensor of $\widetilde{M}$ and $R^{(0,2)}$ the induced Ricci type tensor on $M$ given respectively by

$$
\begin{aligned}
& \widetilde{R i c}(X, Y)=\operatorname{trace}\{Z \rightarrow \widetilde{R}(Z, X) Y\}, \quad \forall X, Y \in \Gamma(T \widetilde{M}), \\
& R^{(0,2)}(X, Y)=\operatorname{trace}\{Z \rightarrow R(Z, X) Y\}, \quad \forall X, Y \in \Gamma(T M) .
\end{aligned}
$$

Consider a quasi-orthonormal frame field $\left\{\xi ; W_{a}\right\}$ on $M$, where $\operatorname{Rad}(T M)=\operatorname{Span}\{\xi\}$ and $S(T M)=\operatorname{Span}\left\{W_{a}\right\}$ and let $E=\left\{\xi, N, W_{a}\right\}$ be the corresponding frame field on $\widetilde{M}$. Using this frame field, we obtain

$$
\begin{aligned}
R^{(0,2)}(X, Y) & =\widetilde{\operatorname{Ric}}(X, Y)+B(X, Y) \operatorname{tr} A_{N}-g\left(A_{N} X, A_{\xi}^{*} Y\right) \\
& -\widetilde{g}(\widetilde{R}(\xi, Y) X, N), \quad \forall X, Y \in \Gamma(T M) .
\end{aligned}
$$

This shows that $R^{(0,2)}$ is not symmetric. The tensor field $R^{(0,2)}$ is called its induced Ricci tensor [5, 6], denoted by Ric, of $M$ if it is symmetric. It is known [13] that $R^{(0,2)}$ is symmetric if and only if the 1 -form $\tau$ is closed, i.e., $d \tau=0$, for any coordinate neighborhood $\mathcal{U} \subset M$.

Remark. If $R^{(0,2)}$ is symmetric, then there exists a null pair $\{\xi, N\}$ such that the corresponding 1-form $\tau$ satisfies $\tau=0$ [4], which called a canonical null pair of $M$. Although $S(T M)$ is not unique, it is canonically isomorphic to the factor vector bundle $S(T M)^{\sharp}=T M / \operatorname{Rad}(T M)$ [17]. This implies that all screen distribution are mutually isomorphic. For this reason, in case $d \tau=0$ we consider only lightlike hypersurfaces $M$ endow with the canonical null pair.

$M$ is called an Einstein manifold if the Ricci tensor of $M$ satisfies

$$
\text { Ric }=\kappa g .
$$

It is well-known that if $\operatorname{dim} M>2$, then $\kappa$ is a constant. For $\operatorname{dim} M=2$, any manifold $M$ is Einstein but $\kappa$ is not necessarily constant.

In case $\widetilde{M}$ is a space form $\widetilde{M}(c), R^{(0,2)}$ is given by

$$
R^{(0,2)}(X, Y)=m c g(X, Y)+B(X, Y) \operatorname{tr} A_{N}-g\left(A_{N} X, A_{\xi}^{*} Y\right) .
$$


Theorem 5.1 [13]. Let $M$ be a lightlike hypersurface of a semi-Riemannian manifold $\widetilde{M}$ admitting a semi-symmetric metric connection. Then the following assertions are equivalent:

(1) The screen distribution $S(T M)$ is an integrable distribution.

(2) $C$ is symmetric, i.e., $C(X, Y)=C(Y, X)$ for all $X, Y \in \Gamma(S(T M))$.

(3) The shape operator $A_{N}$ is self-adjoint with respect to $g$, i.e.,

$$
g\left(A_{N} X, Y\right)=g\left(X, A_{N} Y\right), \quad \forall X, Y \in \Gamma(S(T M)) .
$$

Remark. Just as in the well-known case of locally product Riemannian or semi-Riemannian manifolds $[4,5,6,19]$, if $S(T M)$ is an integrable distribution, then and $M$ is locally a product manifold $\mathcal{C} \times M^{*}$ where $\mathcal{C}$ is a null curve tangent to $\operatorname{Rad}(T M)$ and $M^{*}$ is a leaf of $S(T M)$.

Theorem 5.2. Let $M$ be a screen quasi-conformal Einstein lightlike hypersurface of a Lorentzian space form $\widetilde{M}(c)$ admitting a semi-symmetric non-metric connection. If $\zeta$ is tangent to $M$ but it does not belong to $S(T M)$ and the mean curvature of $M$ is constant, then $M$ is locally a product manifold $M=\mathcal{C} \times M_{1} \times M_{2}$, where $\mathcal{C}$ is a null curve tangent to $\operatorname{Rad}(T M), M_{1}$ is an Euclidean space and $M_{2}$ is a totally umbilical Riemannian space.

Proof. From (3.3), (4.2) and the fact $A_{\xi}^{*}$ is self-adjoint, we show that $R^{(0,2)}$ is symmetric and $S(T M)$ is an integrable distribution. As $g\left(A_{\xi}^{*} \zeta, X\right)$ $=B(\zeta, X)=0$ and $S(T M)$ is non-degenerate, we have

$$
A_{\xi}^{*} \zeta=0 .
$$

Using (2.12), (3.3), (4.1) and the fact $c=1$, from (4.2) we have

$$
g\left(A_{\xi}^{*} X, A_{\xi}^{*} Y\right)-\alpha g\left(A_{\xi}^{*} X, Y\right)+\varphi^{-1}(\kappa-m) g(X, Y)=0,
$$

for all $X, Y \in \Gamma(T M)$ due to $c=1$, where $\alpha=\operatorname{tr} A_{\xi}^{*}-f m \varphi^{-1}$. Taking $X=Y=\zeta$ to (4.4) and using (4.3), we have $\kappa=m$. (4.4) becomes

$$
g\left(A_{\xi}^{*} X, A_{\xi}^{*} Y\right)-\alpha g\left(A_{\xi}^{*} X, Y\right)=0 .
$$

As $\widetilde{M}$ is Lorentzian manifold, $S(T M)$ is a Riemannian vector bundle. Since $\xi$ is an eigenvector field of $A_{\xi}^{*}$ corresponding to the eigenvalue 0 due to $(2.14)_{2}$ and $A_{\xi}^{*}$ is $S(T M)$-valued real self-adjoint operator, $A_{\xi}^{*}$ have $m$ real orthonormal eigenvector fields in $S(T M)$ and is diagonalizable. Consider a frame field of eigenvectors $\left\{\xi, E_{1}, \ldots, E_{m}\right\}$ of $A_{\xi}^{*}$ such that $\left\{E_{1}, \ldots, E_{m}\right\}$ is an orthonormal frame field of $S(T M)$ and $A_{\xi}^{*} E_{i}=\lambda_{i} E_{i}$. 
Put $X=Y=E_{i}$ in (4.5), each eigenvalue $\lambda_{i}$ is a solution of the equation

$$
x^{2}-\alpha x=0 .
$$

As this equation has at most two distinct solutions 0 and $\alpha$, there exists $p \in\{0,1, \ldots, m\}$ such that $\lambda_{1}=\cdots=\lambda_{p}=0$ and $\lambda_{p+1}=\cdots=\lambda_{m}=$ $\alpha(\neq 0)$, by renumbering if necessary. As $\operatorname{tr} A_{\xi}^{*}=0 p+(m-p) \alpha$, we have

$$
(m-p-1) \alpha=f m \varphi^{-1} \text {. }
$$

Consider four distributions $D_{o}, D_{\alpha}, D_{o}^{s}$ and $D_{\alpha}^{s}$ on $S(T M)$ given by

$$
\begin{array}{ll}
D_{o}=\left\{X \in \Gamma(T M) \mid A_{\xi}^{*} X=0\right\}, & D_{o}^{s}=D_{o} \cap S(T M), \\
D_{\alpha}=\left\{U \in \Gamma(T M) \mid A_{\xi}^{*} U=\alpha P U\right\}, & D_{\alpha}^{s}=D_{\alpha} \cap S(T M) .
\end{array}
$$

Clearly we show that $D_{o} \cap D_{\alpha}=\operatorname{Rad}(T M), D_{o}^{s} \cap D_{\alpha}^{s}=\{0\}$ as $\alpha \neq 0$ and $D_{o}^{s}=P D_{o}, D_{\alpha}^{s}=D_{\alpha}$. In the sequel, we take the vector fields $X, Y \in \Gamma\left(D_{o}\right), U, V \in \Gamma\left(D_{\alpha}\right)$ and $Z, W \in \Gamma(T M)$. Denote $X^{*}=$ $P X, Y^{*}=P Y, U^{*}=P U$ and $V^{*}=P V$. Then $X^{*}, Y^{*} \in \Gamma\left(D_{o}^{s}\right)$ and $U^{*}, V^{*} \in \Gamma\left(D_{\alpha}^{s}\right)$. Since $X^{*}$ and $U^{*}$ are eigenvector fields of the real selfadjoint operator $A_{\xi}^{*}$ corresponding to the different eigenvalues 0 and $\alpha$ respectively, $X^{*} \perp U^{*}$ and $g(X, U)=g\left(X^{*}, U^{*}\right)=0$, that is, $D_{o} \perp_{g} D_{\alpha}$. Also, since $B(X, U)=g\left(A_{\xi}^{*} X, U\right)=0$, we show that $D_{\alpha} \perp_{B} D_{o}$. Since $\left\{E_{i}\right\}_{1 \leq i \leq p}$ and $\left\{E_{a}\right\}_{p+1 \leq a \leq m}$ are vector fields of $D_{o}^{s}$ and $D_{\alpha}^{s}$ respectively and $D_{o}^{s}$ and $D_{\alpha}^{s}$ are mutually orthogonal, we show that $D_{o}^{s}$ and $D_{\alpha}^{s}$ are non-degenerate distributions of rank $p$ and rank $(m-p)$ respectively. Thus $S(T M)$ is decomposed as $S(T M)=D_{\alpha}^{s} \oplus_{\text {orth }} D_{o}^{s}$.

From (4.5), we get $A_{\xi}^{*}\left(A_{\alpha}^{*}-\alpha P\right)=0$. Let $W \in \operatorname{Im} A_{\xi}^{*}$. Then there exists $Z \in \Gamma(T M)$ such that $W=A_{\xi}^{*} Z$. Then $\left(A_{\xi}^{*}-\alpha P\right) W=0$ and $W \in \Gamma\left(D_{\alpha}\right)$. Thus $\operatorname{Im} A_{\xi}^{*} \subset \Gamma\left(D_{\alpha}\right)$. By duality, $\operatorname{Im}\left(A_{\xi}^{*}-\alpha P\right) \subset \Gamma\left(D_{o}\right)$.

Applying $\nabla_{X}$ to $B(Y, U)=0$ and using (2.12), we obtain

$$
\left(\nabla_{X} B\right)(Y, U)=-g\left(A_{\xi}^{*} \nabla_{X} Y, U\right) .
$$

Using this, (2.11), (3.6) and the facts $A_{\xi}^{*} X=A_{\xi}^{*} Y=0$, we get

$$
g\left(A_{\xi}^{*}[X, Y], U\right)=0 .
$$

As $\operatorname{Im} A_{\xi}^{*} \subset \Gamma\left(D_{\alpha}\right)$ and $D_{\alpha}$ is non-degenerate, $A_{\xi}^{*}[X, Y]=0$. Thus $[X, Y] \in \Gamma\left(D_{o}\right)$ and $D_{o}$ is integrable. This result implies $\left[X^{*}, Y^{*}\right] \in$ $\Gamma\left(D_{o}\right)$. On the other hand, since $S(T M)$ is integrable, $\left[X^{*}, Y^{*}\right] \in$ $\Gamma(S(T M))$. Thus $\left[X^{*}, Y^{*}\right] \in \Gamma\left(D_{o}^{s}\right)$. Thus $D_{o}^{s}$ is also integrable.

Applying $\nabla_{V}$ to $B(U, Y)=0$ and using $A_{\xi}^{*} Y=0$ and $A_{\xi}^{*} U=\alpha P U$, we get

$$
\left(\nabla_{V} B\right)(U, Y)=-\alpha g\left(\nabla_{V} Y, U\right) .
$$


Substituting this into (3.6) and using the fact $\alpha \neq 0$, we obtain

$$
g\left(\nabla_{V} Y, U\right)=g\left(V, \nabla_{U} Y\right) .
$$

Applying $\nabla_{V}$ to $g(Y, U)=0$ and using (2.10), we have

$$
\pi(Y) g(U, V)-B(V, U) \eta(Y)-g\left(\nabla_{V} Y, U\right)=g\left(Y, \nabla_{V} U\right) .
$$

Taking the skew-symmetric part of this and using (2.11), we have

$$
g([V, U], Y)=0, \quad \forall Y \in \Gamma\left(D_{o}\right) \text { and } U, V \in \Gamma\left(D_{\alpha}\right) .
$$

From this, we get $g\left(\left[V^{*}, U^{*}\right], Y^{*}\right)=0$ for all $Y^{*} \in \Gamma\left(D_{o}^{s}\right)$ and $U^{*}, V^{*} \in$ $\Gamma\left(D_{\alpha}^{s}\right)$. As $D_{o}^{s}$ and $D_{\alpha}^{s}$ are mutually orthogonal non-degenerate distributions, we show that $\left[V^{*}, U^{*}\right] \in \Gamma\left(D_{\alpha}^{S}\right)$. Thus $D_{\alpha}^{s}$ is also integrable.

Applying $\nabla_{U}$ to $B(X, Y)=0$ and $\nabla_{X}$ to $B(U, Y)=0$, we have

$$
\left(\nabla_{U} B\right)(X, Y)=0, \quad\left(\nabla_{X} B\right)(U, Y)=-\alpha g\left(\nabla_{X} Y, U\right) .
$$

Substituting these equations into (3.6), we have $\alpha g\left(\nabla_{X} Y, U\right)=0$. As

$$
g\left(A_{\xi}^{*} \nabla_{X} Y, U\right)=B\left(\nabla_{X} Y, U\right)=\alpha g\left(\nabla_{X} Y, U\right)=0
$$

and $\operatorname{Im} A_{\xi}^{*} \subset \Gamma\left(D_{\alpha}\right)$ and $D_{\alpha}$ is non-degenerate, we get $A_{\xi}^{*} \nabla_{X} Y=0$. This implies $\nabla_{X} Y \in \Gamma\left(D_{o}\right)$. Thus $D_{o}$ is an auto-parallel distribution on $S(T M)$. This implies that $\nabla_{X^{*}} Y^{*} \in \Gamma\left(D_{o}\right)$ for any $X^{*}, Y^{*} \in \Gamma\left(D_{o}^{s}\right)$. As $C\left(X^{*}, Y^{*}\right)=\varphi B\left(X^{*}, Y^{*}\right)+\eta\left(X^{*}\right) \pi\left(Y^{*}\right)=0$, we have $\nabla_{X^{*}} Y^{*}=$ $\nabla_{X^{*}}^{*} Y^{*} \in \Gamma(S(T M))$. Thus $\nabla_{X^{*}} Y^{*} \in \Gamma\left(D_{o}^{s}\right)$ and $D_{o}^{s}$ is also an autoparallel distribution.

As $A_{\xi}^{*} \zeta=0, \zeta$ belongs to $D_{o}$. Thus $\pi(U)=0$ for any $U \in \Gamma\left(D_{\alpha}\right)$. Applying $\nabla_{X}$ to $g(U, Y)=0$ and using (2.10) and the fact $D_{o}$ is autoparallel, we get $g\left(\nabla_{X} U, Y\right)=0$. This implies $\nabla_{X} U \in \Gamma\left(D_{\alpha}\right)$.

Assume that the mean curvature vector field

$$
\mu=\frac{1}{m} g\left(A_{\xi}^{*} E_{a}, E_{a}\right)=\frac{m-p}{m} \alpha
$$

of $M$ is constant. Then $\alpha$ is a constant. Applying $\nabla_{X}$ to $B(U, V)=$ $\alpha g(U, V)$ and $\nabla_{U}$ to $B(X, V)=0$, we have

$$
\left(\nabla_{X} B\right)(U, V)=0, \quad\left(\nabla_{U} B\right)(X, V)=-\alpha g\left(\nabla_{U} X, V\right) .
$$

Substituting this two equations into (3.6) and using $D_{o} \perp_{B} D_{\alpha}$, we have

$$
g\left(\nabla_{U} X, V\right)=\pi(X) g(U, V) .
$$

Applying $\nabla_{U}$ to $g(X, V)=0$ and using (2.10), we obtain

$$
g\left(X, \nabla_{U} V\right)=0 .
$$


From this, we get $g\left(X^{*}, \nabla_{U^{*}} V^{*}\right)=0$ for all $X^{*} \in \Gamma\left(D_{o}^{s}\right)$ and $U^{*}, V^{*} \in$ $\Gamma\left(D_{\alpha}^{s}\right)$. As $D_{o}^{s}$ and $D_{\alpha}^{s}$ are mutually orthogonal non-degenerate distributions, $\nabla_{U^{*}} V^{*} \in \Gamma\left(D_{\alpha}^{S}\right)$ and $D_{\alpha}^{s}$ is auto-parallel distribution.

Since the leaf $M^{*}$ of $S(T M)$ is a Riemannian manifold and $S(T M)=$ $D_{\alpha}^{s} \oplus_{\text {orth }} D_{o}^{s}$, where $D_{\alpha}^{s}$ and $D_{o}^{s}$ are auto-parallel distributions of $M^{*}$, by the decomposition theorem of de Rham [3] we have $M^{*}=M_{1} \times M_{2}$, where $M_{1}$ is a totally geodesic leaf of $D_{o}^{s}$ and $M_{2}$ is a totally umbilical leaf of $D_{\alpha}^{s}$. Consider the frame field of eigenvectors $\left\{\xi, E_{1}, \ldots, E_{m}\right\}$ of $A_{\xi}^{*}$ such that $\left\{E_{i}\right\}_{i}$ is an orthonormal frame field of $S(T M)$, then $B\left(E_{i}, E_{j}\right)=C\left(E_{i}, E_{j}\right)=0$ for $1 \leq i<j \leq m$ and $B\left(E_{i}, E_{i}\right)=$ $C\left(E_{i}, E_{i}\right)=0$ for $1 \leq i \leq m-1$. From (2.15) and (2.19), we have $\widetilde{g}\left(\widetilde{R}\left(E_{i}, E_{j}\right) E_{j}, E_{i}\right)=g\left(R^{*}\left(E_{i}, E_{j}\right) E_{j}, E_{i}\right)=0$. Thus the sectional curvature $K$ of the leaf $M^{\natural}$ of $D_{o}^{s}$ is given by

$$
K\left(E_{i}, E_{j}\right)=\frac{g\left(R^{*}\left(E_{i}, E_{j}\right) E_{j}, E_{i}\right)}{g\left(E_{i}, E_{i}\right) g\left(E_{j}, E_{j}\right)-g^{2}\left(E_{i}, E_{j}\right)}=0 .
$$

Thus $M$ is locally a product manifold $M=\mathcal{C} \times M_{1} \times M_{2}$, where $\mathcal{C}$ is a null curve tangent to $\operatorname{Rad}(T M), M_{1}$ is an Euclidean space and $M_{2}$ is a totally umbilical Riemannian space.

\section{References}

[1] Ageshe, N.S. and Chafle, M.R.: A semi-symmetric non-metric connection on a Riemannian manifold, Indian J. Pure Appl. Math. 23(6) (1992), 399-409.

[2] Călin, C.: Contributions to geometry of CR-submanifold, Thesis, University of Iasi (Romania, 1998).

[3] de Rham, G.: Sur la réductibilité d'un espace de Riemannian, Comm. Math. Helv. 26 (1952), 328-344.

[4] Duggal, K.L. and Bejancu, A.: Lightlike Submanifolds of Semi-Riemannian Manifolds and Applications, Kluwer Acad. Publishers, Dordrecht, 1996.

[5] Duggal, K.L. and Jin, D.H.: Null curves and Hypersurfaces of Semi-Riemannian Manifolds, World Scientific, 2007.

[6] Duggal, K.L. and Jin, D.H.: A Classification of Einstein lightlike hypersurfaces of a Lorentzian space form, J. Geom. Phys. 60 (2010), 1881-1889.

[7] Duggal, K.L. and Sahin, B.: Lightlike Submanifolds of indefinite Sasakian manifolds, Int. J. Math. Math. Sci., 2007, Art ID 57585, 1-21.

[8] Duggal, K.L. and Sahin, B.: Generalized Cauchy-Riemann lightlike Submanifolds of indefinite Sasakian manifolds, Acta Math. Hungar. 122(1-2) (2009), 45-58.

[9] Duggal, K.L. and Sahin, B.: Differential geometry of lightlike submanifolds, Frontiers in Mathematics, Birkhäuser, 2010.

[10] Hawking, S.W. and Ellis, G.F.R.: The large scale structure of space-time, Cambridge University Press, Cambridge, 1973. 
[11] Jin, D.H.: Geometry of lightlike hypersurfaces of a semi-Riemannian space form with a semi-symmetric non-metric connection, submitted in Indian J. Pure Appl. Math.

[12] Jin, D.H.: Einstein lightlike hypersurfaces of a Lorentz space form with a semisymmetric non-metric connection, accepted in Bull. Korean Math. Soc. 2012.

[13] Jin, D.H.: Two characterization theorems for irrotational lightlike geometry, accepted in Commun. Korean Math. Soc. 2013.

[14] Jin, D.H.: Einstein half lightlike submanifolds of a Lorentzian space form with a semi-symmetric non-metric connection, accepted in Journal of Inequalities and Applications, 2013.

[15] Jin, D.H.: Lightlike submanifolds of a semi-Riemannian manifold with a semisymmetric non-metric connection, J. Korean Soc. Math. Edu. Ser. B: Pure Appl. Math. 19(3) (2012), 211-228.

[16] Kang, T.H., Jung, S.D., Kim, B.H., Pak, H.K. and Pak, J.S.: Lightlike hypersurfaces of indefinite Sasakian manifolds, Indian J. Pure Appl. Math. 34 (2003), 1369-1380.

[17] Kupeli, D.N.: Singular Semi-Riemannian Geometry, Kluwer Academic, 366, 1996.

[18] Massamba, F.: Screen almost conformal lightlike geometry in indefinite Kenmotsu space forms, Int. Electron. J. Geom. 5(2) (2012), 36-58.

[19] O'Neill, B., Semi-Riemannian Geometry with Applications to Relativity, Academic Press, 1983.

[20] Yasar, E., Cöken, A.C. and Yücesan, A.: Lightlike hypersurfaces in semiRiemannian manifold with semi-symmetric non-metric connection, Math. Scand. 102 (2008), 253-264.

\section{Dae Ho Jin}

Department of Mathematics, Dongguk University, Gyeongju 780-714, Republic of Korea.

E-mail: jindh@dongguk.ac.kr 